\title{
Derived neutrophil to lymphocyte ratio and monocyte to lymphocyte ratio may be better biomarkers for predicting overall survival of patients with advanced gastric cancer
}

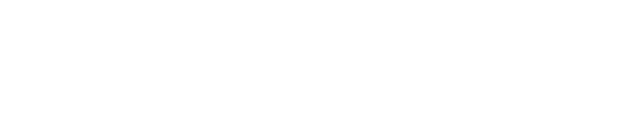

\section{Shubin Song \\ Chunfeng Li \\ Sen Li \\ Hongyu Gao \\ Xiuwen Lan \\ Yingwei Xue}

Department of Gastrointestinal Surgery, Harbin Medical University Cancer Hospital, Harbin, People's Republic of China
Correspondence: Yingwei Xue Department of Gastrointestinal Surgery, Harbin Medical University Cancer Hospital, Haping Road, Harbin, People's Republic of China

$\mathrm{Tel}+8645$ I 86298932

Fax+86 45I86298932

Emailxyw801@163.com
Background and objectives: Preoperative systemic inflammatory response and nutritional status play important roles in the tumorigenesis, progression, and prognosis of gastric cancer (GC). This research is designed to investigate the prognostic value of the biomarkers including the neutrophil to lymphocyte ratio (NLR), derived neutrophil to lymphocyte ratio (dNLR), monocyte to lymphocyte ratio (MLR), platelet to lymphocyte ratio (PLR), and prognostic nutritional index (PNI) in predicting overall survival in patients with GC.

Methods: A total of 1,990 consecutive GC patients who underwent gastrectomy from 2007 to 2011 were enrolled and divided into high level and low level based on the optimal cut-off points for NLR, dNLR, MLR, PLR, and PNI, respectively. The clinicopathological characteristics of the two levels were comparatively analyzed. Overall survival analysis was executed using these biomarkers and clinicopathological characteristics.

Results: The number of metastatic lymph nodes, distant metastasis, American Joint Committee on Cancer TNM stage, radicality, tumor size, metastatic lymph nodes ratio, ascites, and $\mathrm{Hb}$ were all significantly associated with NLR, dNLR, MLR, PLR, and PNI. All of these five biomarkers were closely associated with overall survival in univariate analyses, but only dNLR and MLR were significant in multivariate model. dNLR and MLR can be bonded to predict survival, but whether separate or together, dNLR and MLR were mainly significant in advanced stages.

Conclusion: Although preoperative NLR, dNLR, MLR, PLR, and PNI in peripheral blood proved significant prediction of prognoses of postoperative GC patients, dNLR and MLR may be better biomarkers for predicting overall survival, especially in advanced GC patients.

Keywords: gastric cancer, prognosis, survival, biomarker, systemic inflammatory response

\section{Introduction}

Gastric cancer (GC) is a very common malignant tumor throughout the world, and it leads to cancer-related mortality rates that are higher than those of many other tumors. ${ }^{1}$ Although efforts toward early diagnoses and treatment for GC have made great progress, and we can frequently perform radical surgery for GC, the prognoses for patients with advanced GC are still very poor, and their 5-year survival rate remains in an unsatisfactory range of $10 \%-15 \% .^{2}$ To improve the treatment of GC patients, developing prognostic indicators is critical for improving therapeutic decision-making.

Several lines of research have reported that the immune system plays a crucial role in controlling tumor growth, and neutrophils, lymphocytes, monocytes, and platelets are important for the tumor-induced systemic inflammatory response (SIR). ${ }^{3,4}$ The SIR 
may accelerate tumor development and distant metastases through several mechanisms, such as promoting secretion of inflammatory mediators and cytokines, inhibiting the apoptosis, and damaging the DNA of tumor cells. ${ }^{5}$ Biochemical markers, neutrophils, lymphocytes, monocytes, and platelets can be used to evaluate the host antitumor immune responses and effectively predict cancer prognoses. ${ }^{3}$

In addition, some reports indicate that a "prognostic nutritional index" (PNI) derived from preoperative blood is a gauge of nutritional status that is also associated with the mortality of GC patients. ${ }^{6}$ In this study, we sought clinicopathological characteristics that affected these biomarkers and investigated the relationship of these biochemical markers to the survival of GC patients.

\section{Materials and methods Patients}

A total of 1,990 consecutive patients with histologically proven GC patients, aged 19-88 (mean age: 62 years), were recruited as subjects for this study. They had gastrectomies performed in the Harbin Medical University Cancer Hospital (Heilongjiang, People's Republic of China) between January 2007 and December 2011. All of these patients had preoperative pathological diagnoses through electronic gastroscopies, and the pathological staging was based on the 7 th edition of the TNM-classification given by the Union for International Cancer Control/American Joint Committee on Cancer (UICC/AJCC). Patients were not allowed to eat or drink after 10 PM on the first day they were admitted to hospital, and blood samples were acquired before $6 \mathrm{AM}$ the next day and sent to the clinical laboratory for immediate analysis of standard clinical tests. The inclusion criteria were as follows: 1) All patients who underwent total or subtotal gastrectomy, 2) radical surgery (R0 with clear margins) patients underwent $\mathrm{D} 2+$ lymph nodes resection, while R1 or R2 surgery patients (with residual cancer postoperatively) did not. 3) None of these patients received preoperative neoadjuvant chemotherapy, radiotherapy, or any other antitumor therapies. 4) None of these patients died during a perioperative and a postoperative follow-up time that was longer than 2 months. 5) None of these patients received transfusions before blood tests and none were infected. 6) All of the patients died of GC or GC-related diseases. 7) All of the patients signed written informed consent on the day they were admitted to the hospital to allow the use of their data for any future study. The research project was approved by the Medical Ethics Committee of the Harbin Medical University Cancer Hospital. The clinical characteristics of the 1,990 study subjects with GC are summarized in Table 1.
Table I Characteristic of GC patients

\begin{tabular}{|c|c|}
\hline Variable & $\mathbf{N}(\%)$ \\
\hline \multicolumn{2}{|l|}{ Sex } \\
\hline Female & $523(26.3)$ \\
\hline Male & $\mathrm{I}, 467$ (73.7) \\
\hline \multicolumn{2}{|l|}{ Age (year) } \\
\hline$\leq 62$ & $1,232(61.9)$ \\
\hline$>62$ & $758(38.1)$ \\
\hline \multicolumn{2}{|l|}{ Tumor depth } \\
\hline $\mathrm{TI}$ & $124(6.2)$ \\
\hline $\mathrm{T} 2$ & $187(9.4)$ \\
\hline T3 & $512(25.7)$ \\
\hline $\mathrm{T} 4$ & $\mathrm{I}, \mathrm{I} 67$ (58.7) \\
\hline \multicolumn{2}{|l|}{ Lymph nodes } \\
\hline No & $460(23.1)$ \\
\hline NI & $343(17.2)$ \\
\hline N2 & $447(22.5)$ \\
\hline N3 & $740(37.2)$ \\
\hline \multicolumn{2}{|l|}{ Metastasis } \\
\hline Mo & $\mathrm{I}, 78 \mathrm{I}(89.5)$ \\
\hline MI & $209(10.5)$ \\
\hline \multicolumn{2}{|l|}{ AJCC stage } \\
\hline I & $162(8.1)$ \\
\hline ॥ & $476(23.9)$ \\
\hline III & $\mathrm{I}, \mathrm{I} 43(57.5)$ \\
\hline IV & $209(10.5)$ \\
\hline \multicolumn{2}{|l|}{ Radicality } \\
\hline RO & $\mathrm{I}, 468(73.8)$ \\
\hline $\mathrm{R} 1$ or $\mathrm{R} 2$ & $522(26.2)$ \\
\hline \multicolumn{2}{|l|}{ Tumor size $(\mathrm{cm})$} \\
\hline$\leq 6$ & $\mathrm{I}, 290(64.8)$ \\
\hline$>6$ & $700(35.2)$ \\
\hline \multicolumn{2}{|l|}{ Location } \\
\hline Upper & $273(13.7)$ \\
\hline Middle & $37 \mid(18.6)$ \\
\hline Low & $\mathrm{I}, 160(58.3)$ \\
\hline Whole & $186(9.4)$ \\
\hline \multicolumn{2}{|l|}{ MLNR $^{\mathrm{a}}$} \\
\hline$\leq 31.5 \%$ & $\mathrm{I}, \mathrm{I} 55$ (58.0) \\
\hline$>31.5 \%$ & $835(42.0)$ \\
\hline \multicolumn{2}{|l|}{ Differentiation ${ }^{\mathrm{b}}$} \\
\hline Differentiated & $322(16.2)$ \\
\hline Undifferentiated & $\mathrm{I}, 668(83.8)$ \\
\hline \multicolumn{2}{|l|}{ Ascites } \\
\hline No & $1,912(96.1)$ \\
\hline Yes & $78(3.9)$ \\
\hline \multicolumn{2}{|l|}{ CAI9-9 (U/mL) } \\
\hline$\leq 37$ & $\mathrm{I}, 556(78.2)$ \\
\hline$>37$ & $434(21.8)$ \\
\hline \multicolumn{2}{|l|}{ CEA (ng/mL) } \\
\hline$\leq 5$ & $\mathrm{I}, 527(76.7)$ \\
\hline$>5$ & $463(23.3)$ \\
\hline \multicolumn{2}{|l|}{$\mathrm{Hb}(\mathrm{g} / \mathrm{L})$} \\
\hline$\leq 130$ & $\mathrm{I}, 0 \mathrm{I} 4(5 \mathrm{I} .0)$ \\
\hline$>130$ & $976(49.0)$ \\
\hline \multicolumn{2}{|l|}{ Tobacco } \\
\hline Yes & $985(49.5)$ \\
\hline No & $\mathrm{I}, 005(50.5)$ \\
\hline
\end{tabular}


Table I (Continued)

\begin{tabular}{ll}
\hline Variable & $\mathbf{N}(\%)$ \\
\hline dNLR & \\
$\quad \leq 1.73$ & $1,206(60.6)$ \\
$>1.73$ & $784(39.4)$ \\
MLR & \\
$\quad \leq 0.22$ & $917(46.1)$ \\
$>0.22$ & $1,073(53.9)$ \\
NLR & \\
$\quad \leq 2.10$ & $1,018(51.2)$ \\
$>2.10$ & $972(48.8)$ \\
PLR & \\
$\leq 139.12$ & $908(45.6)$ \\
$>139.12$ & $1,082(54.4)$ \\
PNI & \\
$\leq 51.07$ & $960(48.2)$ \\
$>51.07$ & $1,030(51.8)$ \\
\hline
\end{tabular}

Notes: ${ }^{\mathrm{T} T h e}$ average point of MLNR was $31.5 \%$. ${ }^{\mathrm{G}} \mathrm{Grades} \mathrm{I}$ and 2 were differentiated, and grades 3 and 4 were undifferentiated.

Abbreviations: AJCC, American Joint Committee on Cancer; CEA, carcinoembryonic antigen; CA19-9, carbohydrate antigen 19-9; dNLR, derived neutrophil to lymphocyte ratio; GC, gastric cancer; Hb, hemoglobin; MLNR, Metastatic lymph nodes ratio; MLR, monocyte to lymphocyte ratio; NLR, neutrophil to lymphocyte ratio; PLR, platelet to lymphocyte ratio; PNI, prognostic nutritional index.

Patients underwent relevant investigation 1 week before surgery. All patients recruited with stage IV cancer were confirmed by pathology of their liver, lung, or peritoneal metastases and complications such as bleeding, perforation, and pyloric obstruction. The standard blood tests obtained on the morning after admission included albumin $(\mathrm{g} / \mathrm{L})$, white blood cell count $\left(10^{9} / \mathrm{L}\right)$, neutrophil count $\left(10^{9} / \mathrm{L}\right)$, lymphocyte count $\left(10^{9} / \mathrm{L}\right)$, monocyte count $\left(10^{9} / \mathrm{L}\right)$, platelet count $\left(10^{9} / \mathrm{L}\right)$, carbohydrate antigen 19-9 (CA19-9) $(\mathrm{U} / \mathrm{mL})$, and carcinoembryonic antigen (CEA) $(\mathrm{ng} / \mathrm{mL})$. The neutrophil to lymphocyte ratio (NLR), derived neutrophil to lymphocyte ratio (dNLR), monocyte to lymphocyte ratio (MLR), platelet to lymphocyte ratio (PLR), and prognostic nutritional index (PNI) were calculated using the following formulas: ${ }^{7-9}$

NLR = Neutrophil count/Lymphocyte count;

dNLR $=$ Neutrophil count/(White blood cell count Neutrophil count);

MLR = Monocyte count/Lymphocyte count;

PLR = Platelet count/Lymphocyte count;

$\mathrm{PNI}=$ Albumin count + Lymphocyte count $\times 5$.

\section{Patient follow-up}

Every patient was followed up regularly until June 2016 or death (In the first 2 postoperative years, it was every 3 months, and in the following several years it was at 6 months intervals). The total duration of follow-up varied from 3 months to 9 years, with a median of 37 months. Overall survival time was calculated as the interval from the surgery to death.
Table 2 The optimal cut-off point for overall survival

\begin{tabular}{llll}
\hline Variables & AUC & Cut-off point & P-value \\
\hline NLR & 0.555 & 2.10 & $<0.001$ \\
dNLR & 0.551 & 1.73 & $<0.001$ \\
MLR & 0.554 & 0.22 & $<0.001$ \\
PLR & 0.576 & 139.12 & $<0.001$ \\
PNI & 0.395 & - & $<0.001$ \\
\hline
\end{tabular}

Note: '-' indicates no appropriate cut-off point.

Abbreviations: AUC, area under the curve; NLR, neutrophil to lymphocyte ratio; dNLR, derived neutrophil to lymphocyte ratio; MLR, monocyte to lymphocyte ratio: PLR, platelet to lymphocyte ratio; PNI, prognostic nutritional index.

\section{Statistical analysis}

The optimal cut-off levels for NLR, dNLR, MLR, and PLR were determined by receiver operating curve (ROC) analysis. ${ }^{10,11}$ As the optimal cut-off level for PNI did not have statistical significance, we took the average PNI value (51.07) as the cut-off point (Table 2, Figure 1). $\chi^{2}$ tests were used to compare and assess the association between NLR,

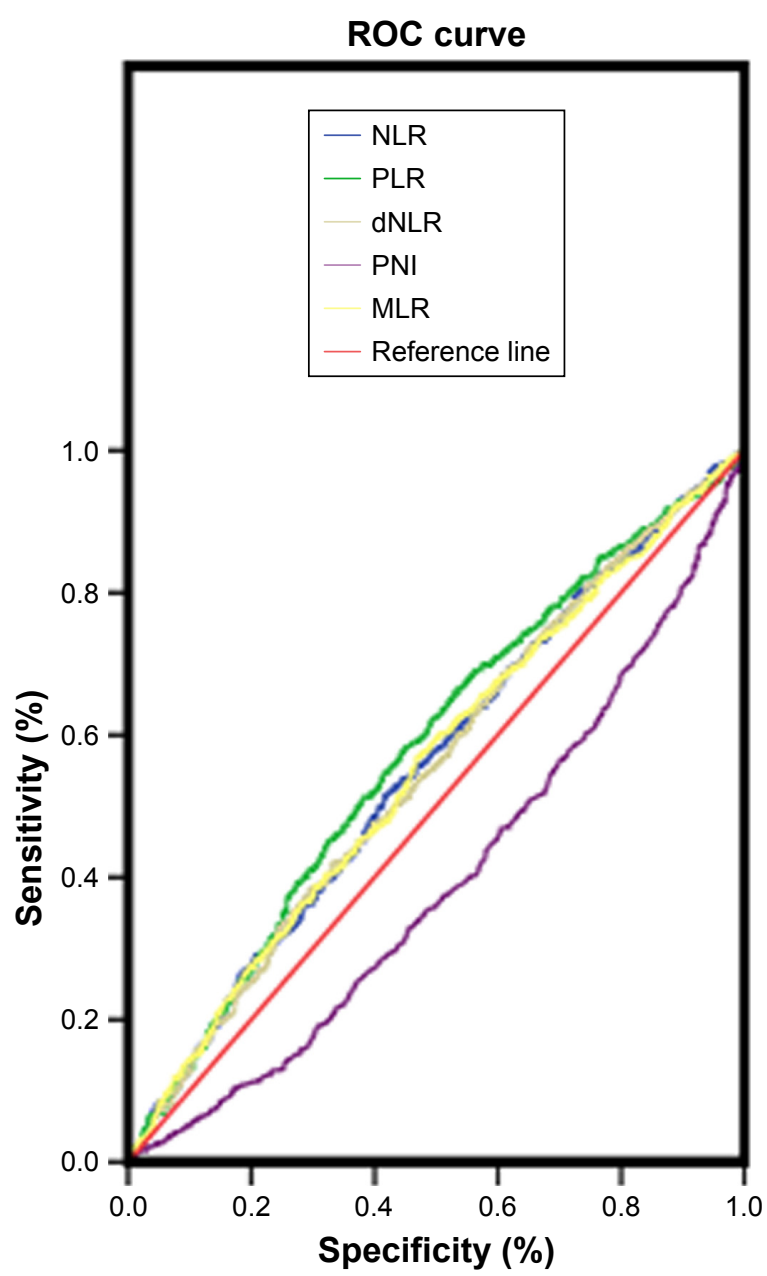

Figure I Optimal cut-off points for NLR, dNLR, MLR, PLR, and PNI were applied with ROC curves.

Abbreviations: $d N L R$, derived neutrophil to lymphocyte ratio; MLR, monocyte to lymphocyte ratio; NLR, neutrophil to lymphocyte ratio; PLR, platelet to lymphocyte ratio; $\mathrm{PNI}$, prognostic nutritional index; ROC, receiver operating characteristics. 
dNLR, MLR, PLR, and PNI and the subjects' clinicopathological characteristics. Survival curves were calculated by the Kaplan-Meier method, and the equivalences of survival curves were analyzed using the log-rank test. Multivariate analysis was evaluated by Cox proportional hazards model, and all of the significant characteristics in univariate analysis were carried into multivariate analysis. A $P<0.05$ was considered statistically significant. All of the statistical analyses were performed using SPSS version 17.0 (IBM Corp., Armonk, NY, USA).

\section{Results}

\section{The relationship between}

\section{clinicopathological characteristics} and biomarkers

We calculated the contrasts between the higher and lower biomarker levels of NLR, dNLR, MLR, PLR, and PNI to study the relationship between the different patients' clinicpathological characteristics and biomarkers (Table 3). We found that NLR was significantly associated with age, tumor invasion (T), lymph nodes metastasis $(\mathrm{N})$, distant metastasis, TNM stage, surgical radicality, tumor size, metastatic lymph node ratio (MLNR), ascites, CEA, CA19-9, and $\mathrm{Hb}$. dNLR was significantly associated with age, lymph nodes metastasis, distant metastasis, stage, radicality, tumor size, MLNR, ascites, CEA, CA19-9, and Hb. MLR was significantly associated with sex, age, tumor invasion, lymph nodes metastasis, distant metastasis, TNM stage, radicality, tumor size, MLNR, ascites, CEA, CA19-9, and Hb. PLR was significantly associated with sex, tumor invasion, lymph nodes metastasis, distant metastasis, TNM stage, radicality, tumor size, MLNR, ascites, CEA, CA19-9, Hb, and smoking. PNI was significantly associated with tumor invasion, lymph nodes metastasis, distant metastasis, TNM stage, radicality, tumor size, MLNR, ascites, and $\mathrm{Hb}$.

Thus, these tumor-related factors such as lymph node metastases, distant metastasis, AJCC TNM stage, radicality, tumor size, MLNR, ascites, and $\mathrm{Hb}$ were all significantly associated with NLR, dNLR, MLR, PLR, and PNI.

\section{The relationship between biomarkers, clinicopathological characteristics, and clinical prognosis}

The results revealed that age $(>62$ years), deeper tumor invasion, more lymph nodes with metastatic involvement, distant metastasis, advanced TNM stage, an R1 or R2 resection (without "clean margins" and leaving residual

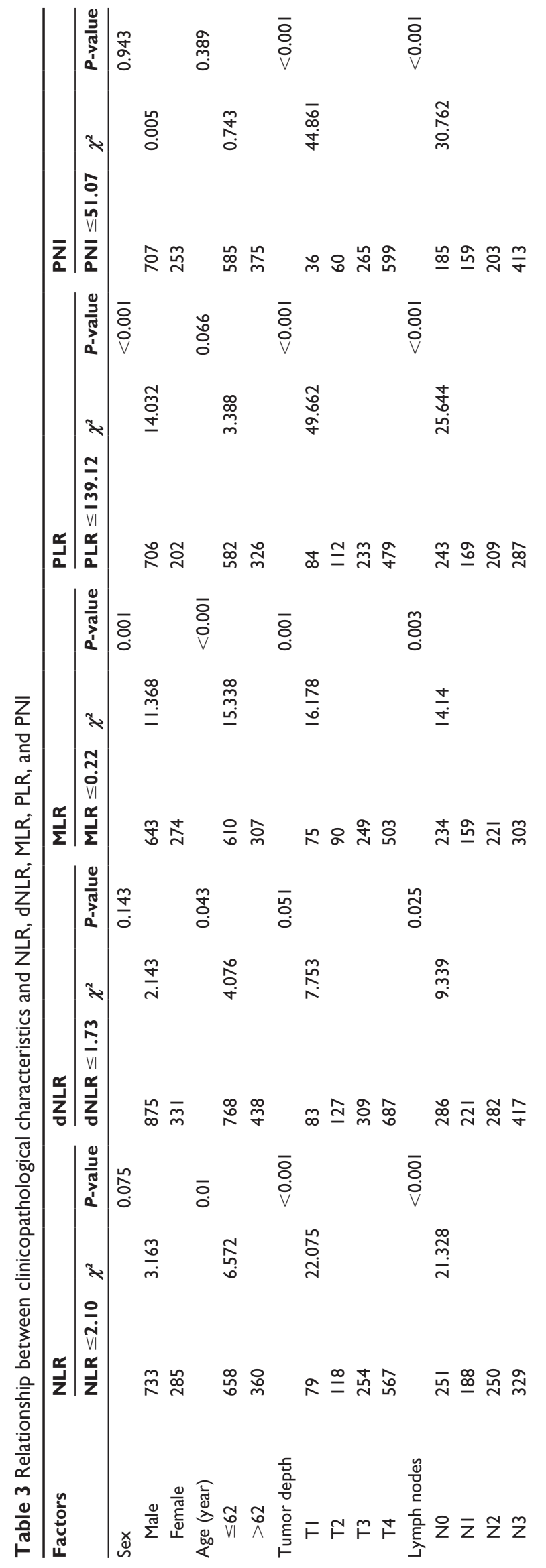




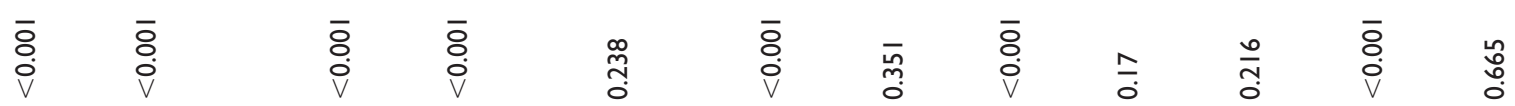

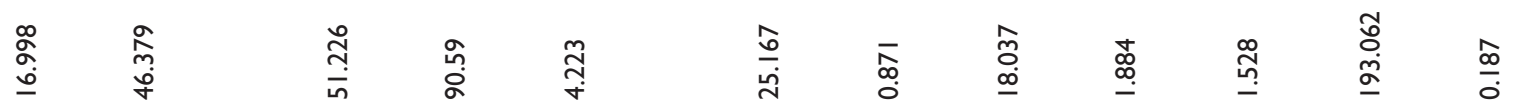

๓

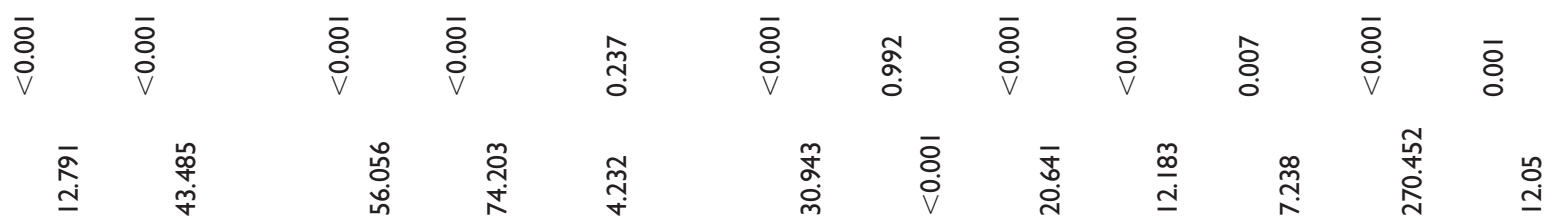

网

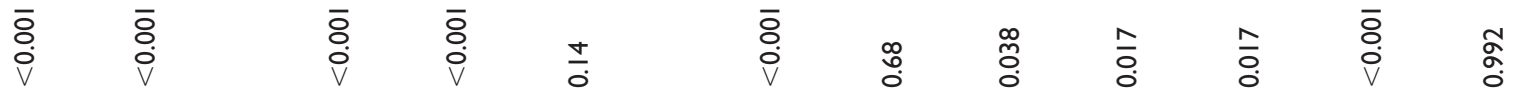

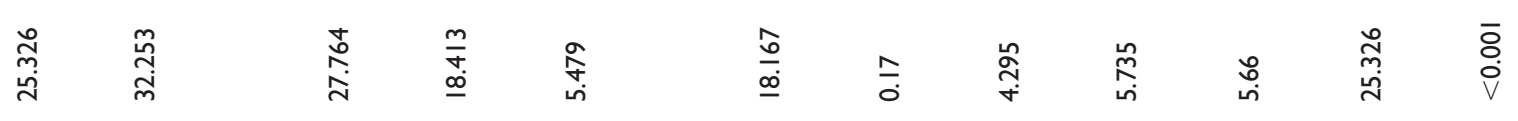
贺 ๙ర

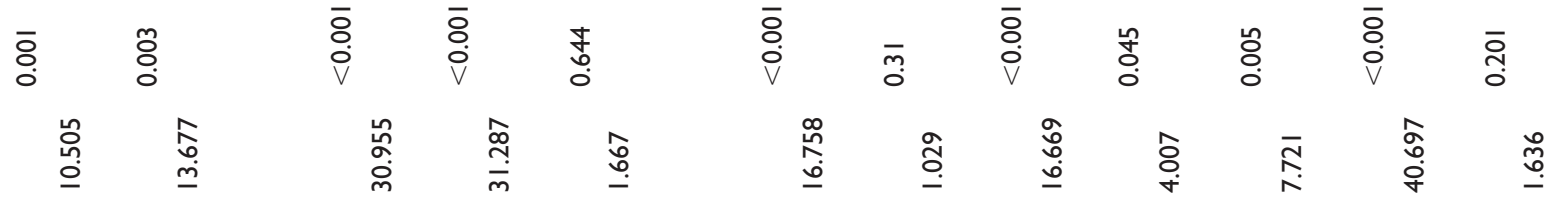

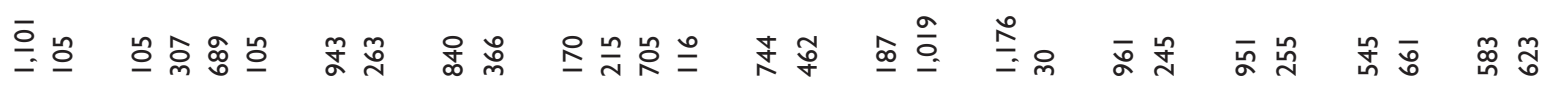

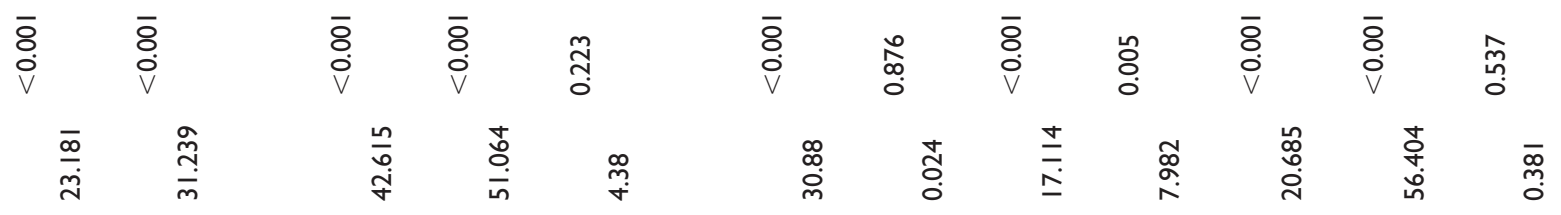

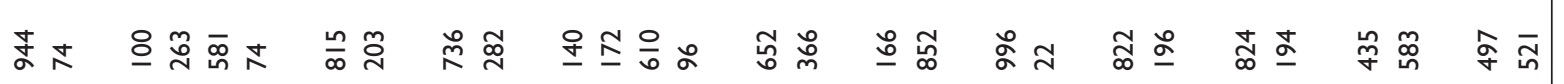

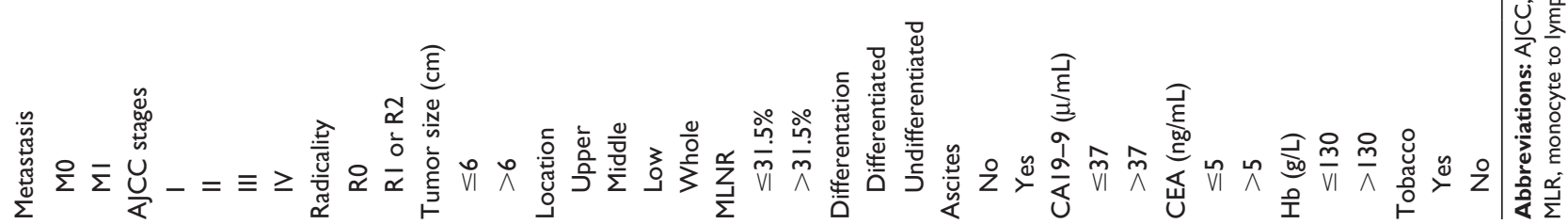


tumor), larger tumor size $(>6 \mathrm{~cm})$, upper tumor location, higher MLNR (>31.5\%), undifferentiated neoplasms, ascites, higher CA19-9 levels ( $>37 \mathrm{U} / \mathrm{mL})$, higher CEA $(>5 \mathrm{ng} / \mathrm{mL})$, lower $\mathrm{Hb}(\leq 130 \mathrm{~g} / \mathrm{L}), \mathrm{NLR}(>2.10)$, dNLR $(>1.73), \operatorname{MLR}(>0.22), \operatorname{PLR}(>139.12)$, and PNI $(\leq 51.07)$ were significantly connected with reduced overall survival time in univariate Kaplan-Meier analyses and log-rank tests (Table 4, Figure 2). The significant factors in the univariate Kaplan-Meier analyses were further studied in multivariate Cox regression model, and our results indicated that age, tumor depth, the number of metastatic lymph nodes, distant metastasis, AJCC TNM stage, radicality, tumor size, MLNR, differentiation, CA19-9, CEA, dNLR, and MLR were significantly associated with overall survival time $(P<0.05$ for all). Thus, dNLR and MLR were independent risk factors for overall survival time (Table 4).

Then, as the long-term clinical outcomes of dNLR $(P<0.001)$ and MLR $(P<0.001)$ were similar to each other, we tried to study the prognostic value of dNLR and MLR combined together in the whole cohort. We hypothesized that patients with $\mathrm{dNLR}>1.73$ and MLR $>0.22$ had scores of 2 , and $\mathrm{dNLR} \leq 1.73$ and MLR $\leq 0.22$ had scores of 0 .

Table 4 Univariate and multivariate analysis of factors for overall survival

\begin{tabular}{|c|c|c|c|}
\hline \multirow[t]{2}{*}{ Factors } & \multirow{2}{*}{$\frac{\text { Univariate }}{P \text {-value }}$} & \multicolumn{2}{|l|}{ Multivariate } \\
\hline & & HR (95\% CI) & $P$-value \\
\hline Sex & 0.836 & & \\
\hline Age (year) & $<0.001$ & I.196 (I.07I-I.336) & 0.002 \\
\hline Tumor depth & $<0.001$ & I.18I (1.066-I.308) & 0.001 \\
\hline Lymph nodes & $<0.001$ & $1.215(1.105-1.336)$ & $<0.001$ \\
\hline Distant metastasis & $<0.001$ & $0.667(0.508-0.875)$ & 0.004 \\
\hline AJCC stage & $<0.001$ & $2.087(1.698-2.566)$ & $<0.001$ \\
\hline Radicality & $<0.001$ & $2.140(1.860-2.463)$ & $<0.001$ \\
\hline Tumor size (cm) & $<0.001$ & $1.311(1.164-1.476)$ & $<0.001$ \\
\hline Tumor location & $<0.001$ & $0.958(0.899-1.020)$ & 0.180 \\
\hline MLNR & $<0.001$ & $1.295(1.104-1.519)$ & 0.001 \\
\hline Differentiation & $<0.001$ & 1.188 (1.018-1.385) & 0.029 \\
\hline Ascites & $<0.001$ & I.24I (0.965-I.595) & 0.092 \\
\hline CAI9-9 $(\mu / \mathrm{mL})$ & $<0.001$ & $1.218(1.076-1.378)$ & 0.002 \\
\hline CEA (ng/mL) & $<0.001$ & $1.182(1.045-1.336)$ & 0.008 \\
\hline $\mathrm{Hb}(\mathrm{g} / \mathrm{L})$ & $<0.001$ & I.004 (0.892-I.I30) & 0.944 \\
\hline Tobacco & 0.376 & & \\
\hline $\mathrm{dNLR}$ & $<0.001$ & $1.202(1.007-1.435)$ & 0.042 \\
\hline MLR & $<0.001$ & $1.156(1.023-1.305)$ & 0.020 \\
\hline NLR & $<0.001$ & $0.86 I(0.7 \mid 4-1.039)$ & 0.119 \\
\hline PLR & $<0.001$ & $0.933(0.820-1.062)$ & 0.291 \\
\hline PNI & $<0.001$ & $0.95 \mid(0.842-1.075)$ & 0.423 \\
\hline
\end{tabular}

Abbreviations: AJCC, American Joint Committee on Cancer; CEA, carcinoembryonic antigen; CA 19-9, carbohydrate antigen 19-9; dNLR, derived neutrophil to lymphocyte ratio; $\mathrm{Hb}$, hemoglobin; HR, hazard ratio; MLNR, metastatic lymph node ratio. MLR, monocyte to lymphocyte ratio; NLR, neutrophil to lymphocyte ratio; $\mathrm{PLR}$, platelet to lymphocyte ratio; PNI, prognostic nutritional index.
Patients with $\mathrm{dNLR}>1.73$ and $\mathrm{MLR} \leq 0.22$ or $\mathrm{dNLR} \leq 1.73$ and MLR $>0.22$ had score of 1 . Figure 3 showed that the overall survival time decreased as the scores increased. We thus found that dNLR combined with MLR could perfectly predict prognoses $(P<0.001)$. Finally, we analyzed the prognostic value of dNLR, MLR, and their combination scores when patients were divided by the AJCC TNM stage. The biomarker dNLR was significant in stages III $(P=0.001)$ and IV $(P=0.004)$, but not in stages I $(P=0.263)$ and II $(P=0.676)$. MLR was significant in stages I $(P=0.038)$ and III $(P=0.010)$, but not in stages II $(P=0.208)$ and IV $(P=0.067)$; the combination score was significant in stages III $(P=0.001)$ and IV $(P=0.015)$, but not in stages I $(P=0.165)$ and II $(P=0.484)$ (Figure 4$)$.

\section{Discussion}

Pietrzyk et al $^{12}$ reported that hematological parameters such as NLR and PLR could be used to discriminate GC patients from non-GC patients. It was also reported that SIR had a close relationship with the prognoses of many tumors. ${ }^{13}$ Inflammation could promote cellular proliferation in neoplasms, stimulate angiogenesis, and lead to lower immunity, thus promoting cancer progression and distant metastases of tumors. ${ }^{14}$ Many clinical studies have shown that the occurrence and development of GC is closely linked with a chronic SIR. ${ }^{7,15,16}$ SIR was also reported to be correlated with chemotherapy responses in patients with unresectable GC. ${ }^{17}$ A few studies showed that PNI as a nutritional status indicator was helpful in predicting survival of patients with GC and many other tumors., ${ }^{9,18,19}$

A higher NLR, dNLR, MLR, PLR, or lower PNI means elevated neutrophils, monocytes, and platelets or decreased lymphocytes and serum albumin. Neutrophils can secret VEGF (vascular endothelial growth factor), ROS (reactive oxygen species), NO (nitric oxide), interleukin-18, and matrix metalloproteinase and can suppress the tumor-induced T-cell response that promotes tumorigenesis, growth, and metastasis. ${ }^{20}$ Platelets can accelerate tumor growth by secreting VEGF, which then promotes angiogenesis. ${ }^{21}$ Lymphocytes, especially $\mathrm{CD} 3^{+} \mathrm{T}$-cells, $\mathrm{CD} 8^{+} \mathrm{T}$-cells, and NK cells, can inhibit tumorigenesis and kill tumor cells efficiently. A decreased lymphocyte count leads to a decreased antitumor response. ${ }^{22,23}$ Macrophages originating from monocytes can devour tumor cells. This anticancer activity can be augmented when chemokines stimulated by the tumor microenvironment promote macrophage chemotaxis to the neoplastic tissues. ${ }^{24}$ While albumin levels are used to assess nutritional status and immune function, lower albumin is 

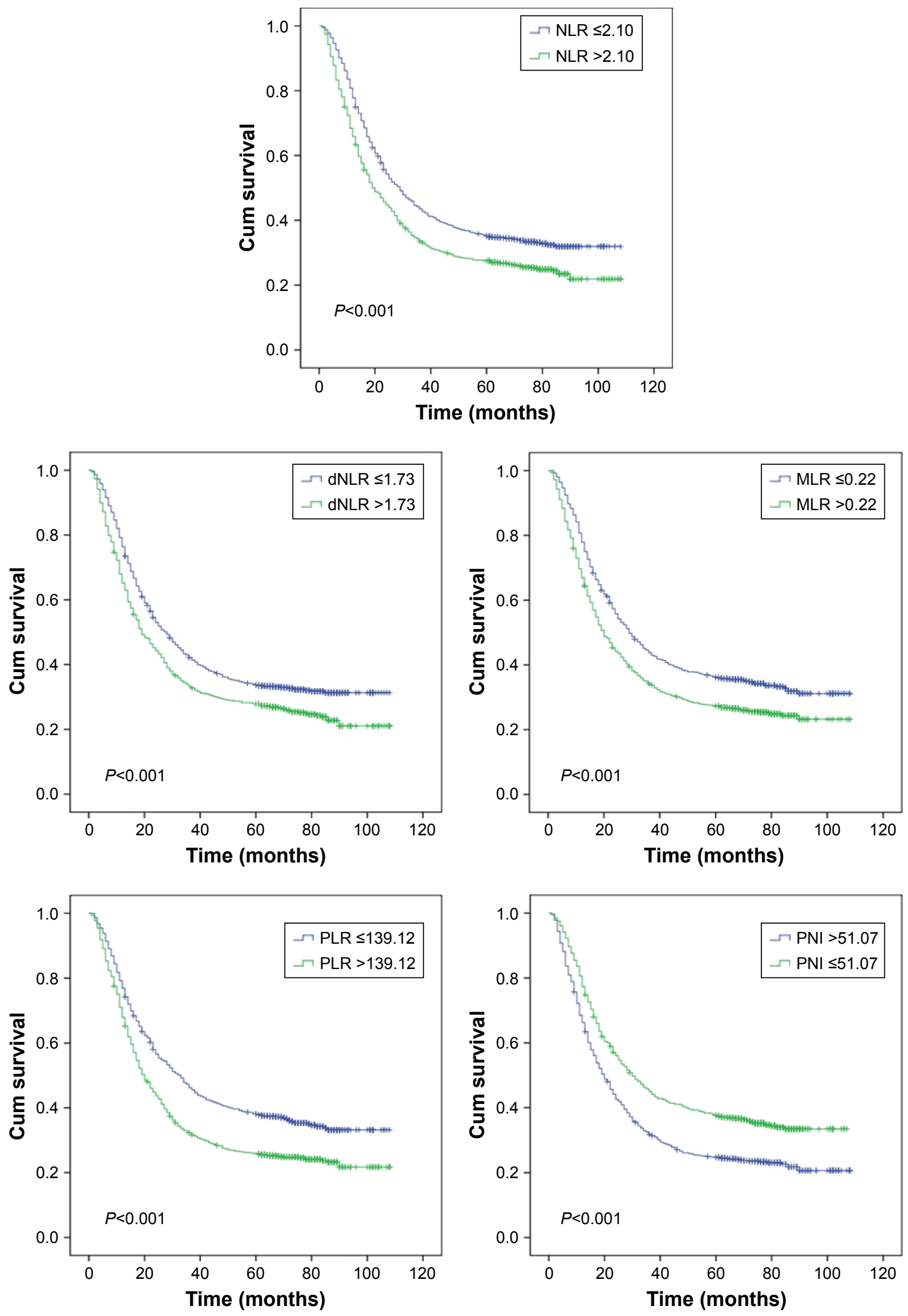

Figure 2 Kaplan-Meier curves for overall survival according to NLR, dNLR, MLR, PLR, and PNI.

Abbreviations: $\mathrm{dNLR}$, derived neutrophil to lymphocyte ratio; MLR, monocyte to lymphocyte ratio; NLR, neutrophil to lymphocyte ratio; PLR, platelet to lymphocyte ratio; $\mathrm{PNI}$, prognostic nutritional index. 


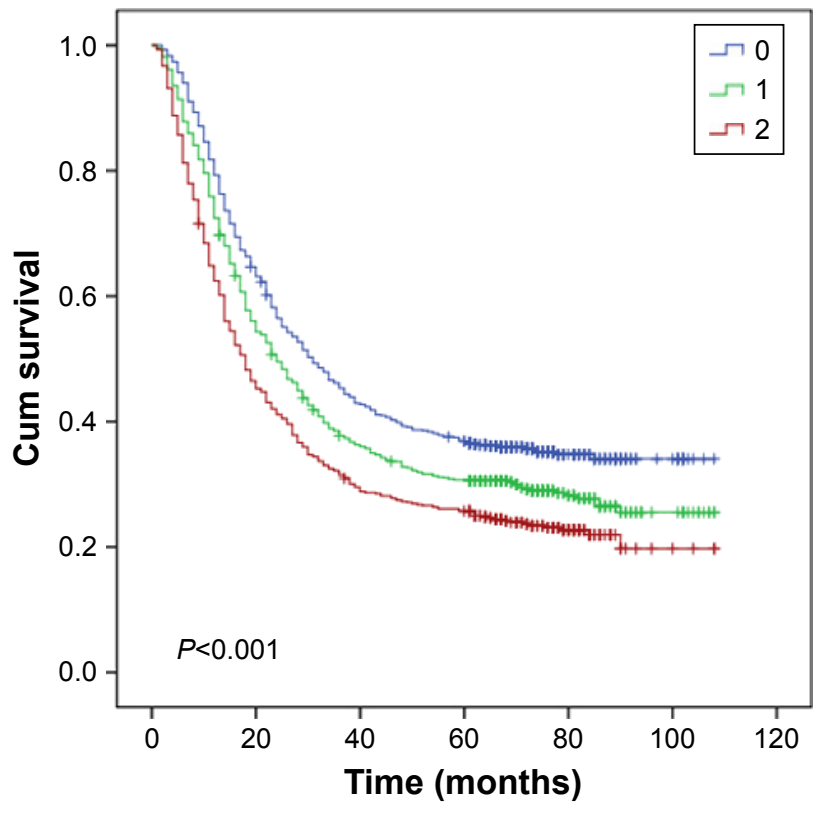

Figure 3 Kaplan-Meier curves for overall survival according to scores of dNLR and MLR.

Abbreviations: dNLR, derived neutrophil to lymphocyte ratio; MLR, monocyte to lymphocyte ratio.

associated with tumor progression, metastasis, and higher risk of death after surgery. ${ }^{25}$

Of the clinical factors such as metastatic lymph nodes, distant metastasis, AJCC TNM stage, radical resection, tumor size, MLNR, ascites, and $\mathrm{Hb}$ that affect NLR, dNLR, MLR, PLR, and PNI simultaneously, only ascites and $\mathrm{Hb}$ were not independent prognostic factors for overall survival. This may suggest that bleeding can lead to increased white blood cell counts and albumin loss that is similar to inflammation and malnutrition, but that this phenomenon was different from the SIR that accompanied tumor appearance and growth. The five biomarkers (NLR, dNLR, MLR, PLR, and PNI) monitored preoperatively in the blood of GC patients have close relationship with the prognoses and overall survival of the patient. Higher NLR, dNLR, MLR, and PLR and lower PNI predicted shorter survival time for GC patients with resectable neoplastic lesions. We have no definite mechanisms to explain these observations, although it has been suggested that this result may be related to the immune microenvironment of the tumor cells. ${ }^{5,26}$

Our findings for NLR, dNLR, MLR, PLR, and PNI are all derived from routine peripheral blood results of preoperative patients, and they are simple and convenient to use for predicting the prognoses and survival of tumor patients. The GC patients need no additional costly investigations to obtain these risk indicators. However, in our study only dNLR and MLR were independent risk factors for overall survival in the Cox multivariate analysis. Kim et $\mathrm{al}^{27}$ found
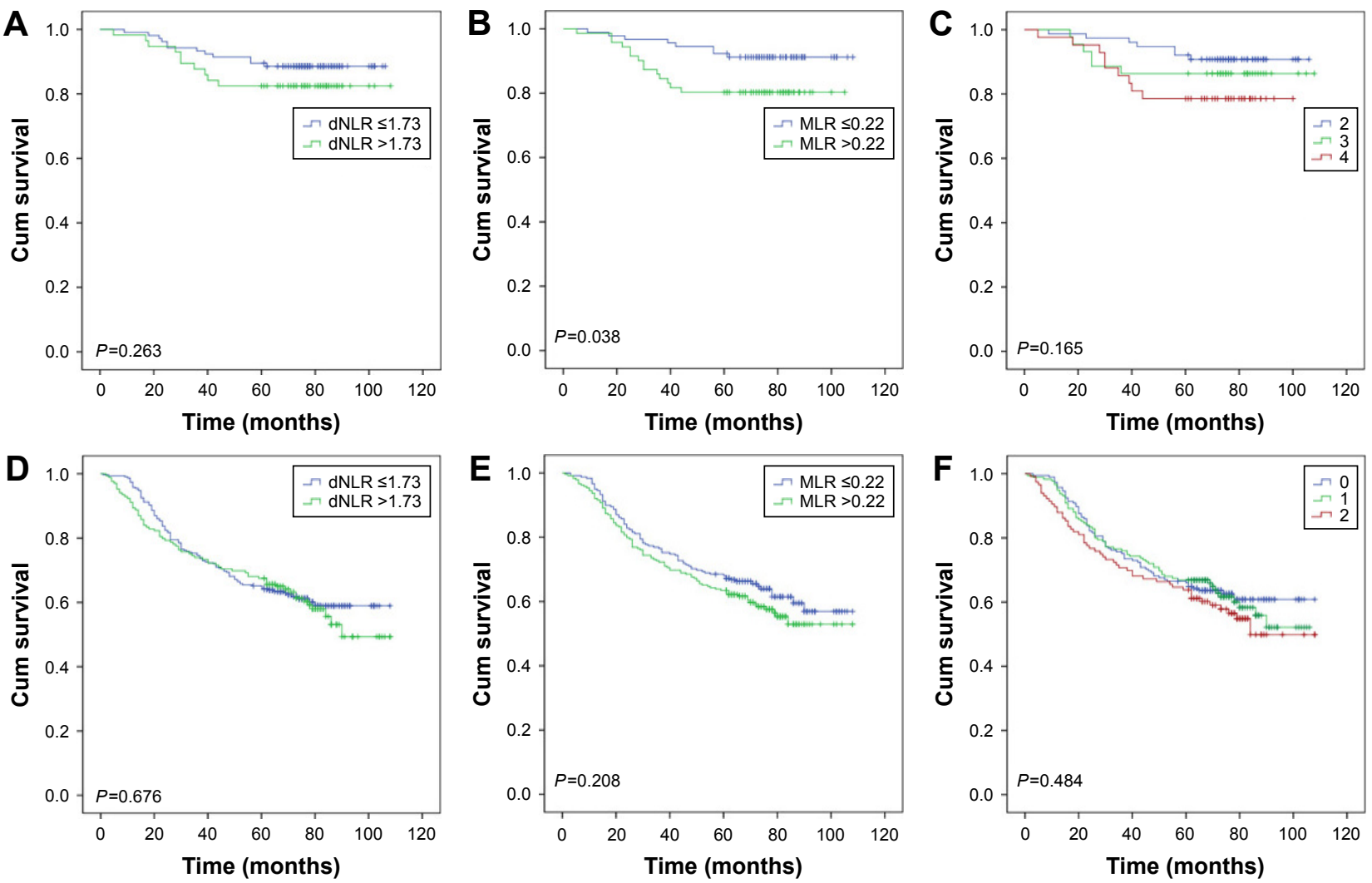

Figure 4 (Continued) 

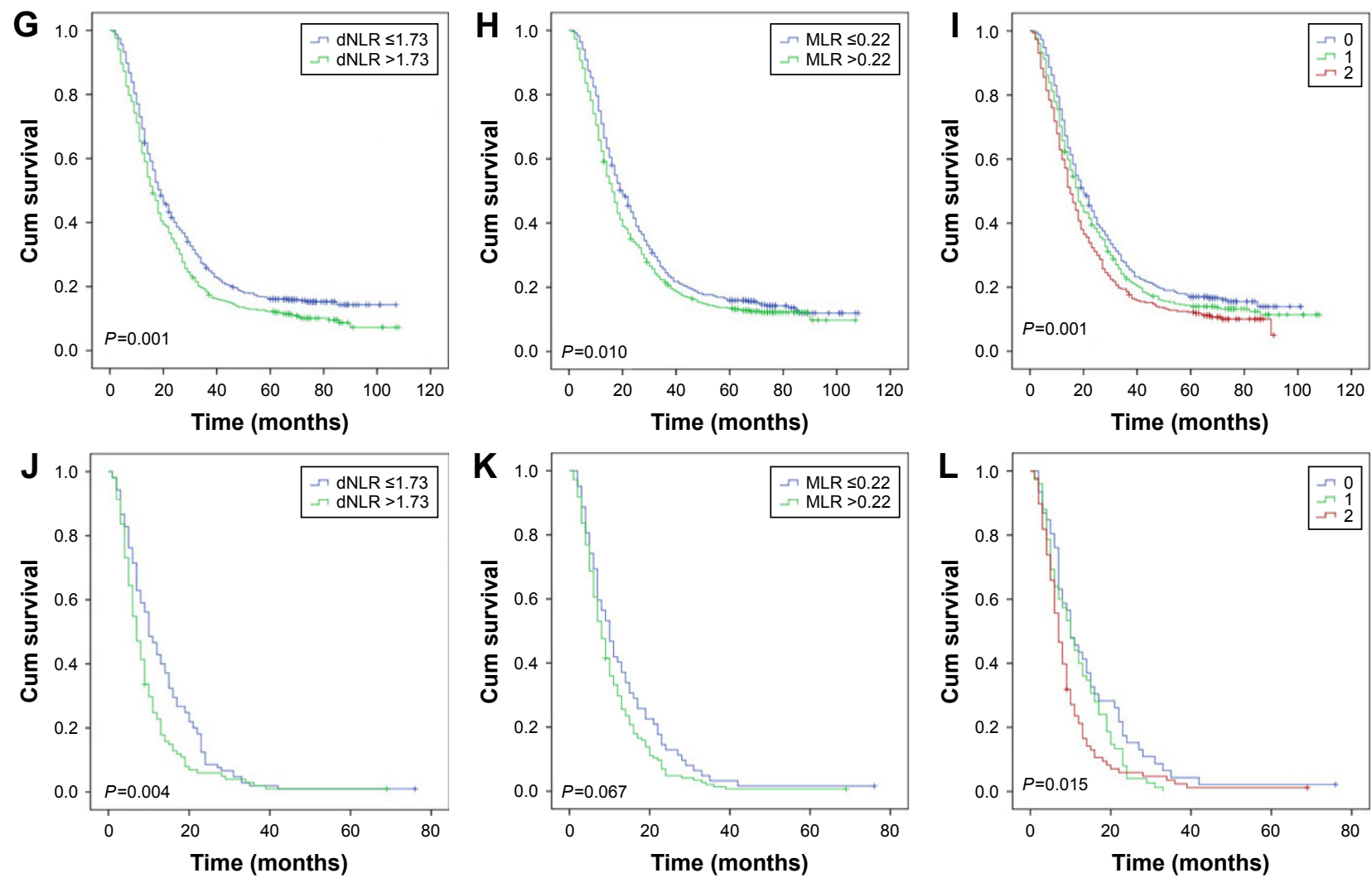

Figure 4 Overall survival stratified by TNM stage of $d N L R, M L R$, and their combination score. A, D, G, and J were stages I, II, III, and IV of dNLR, respectively; B, E, H and K were stages I, II, III, and IV of MLR, respectively; C, F, I, and L were stages I, II, III, and IV of their combination scores.

Abbreviations: $\mathrm{dNLR}$, derived neutrophil to lymphocyte ratio; MLR, monocyte to lymphocyte ratio.

that NLR was a better indicator than PLR for predicting overall survival. Sakurai et $\mathrm{al}^{9}$ found that PNI was effective for predicting overall survival in elderly and stage I GC patients after gastrectomy. In contrast, our present results failed to confirm the prognostic value of NLR, PLR, and PNI in a Cox multivariate analysis. At the same time, we found that dNLR, MLR, and their combination scores were mainly statistically significant in later stages (III and IV), but not in earlier stages of GC, and the combination scores we derived did not show superior results over dNLR or MLR separately. In spite of this, the SIR may still have been becoming more intense as the tumors progressed, but was not obvious in the initial stages. However, further investigations will be needed to illuminate this phenomenon.

A few limitations of this research should be reviewed. First, this was a retrospective study in a single institution, and some patients were lost to follow-up. Second, GC patients with III/IV stage disease account for $68 \%$ of the study population, which may influence the results. Third, some patients with factors such as vascular invasion and postoperative adjuvant chemotherapy, which may affect prognoses to a large extent, were not enrolled according to the study criteria. Therefore, further research will be needed to clarify the relationship between the inflammatory biomarkers and the prognosis of GC patients.

\section{Conclusion}

In summary, preoperative NLR, dNLR, MLR, PLR, and PNI in peripheral blood proved to be significant prognostic indicators of the postoperative course of GC patients. Moreover, dNLR and MLR are independent prognostic factors for overall survival and may be better biomarkers in predicting overall survival of patients with GC, especially those with advanced stages of disease. A broad range of institutions should be organized to perform a multicenter study on the prognostic significance of these indicators. Perhaps, in countries like China, which have less developed economies, these readily available and inexpensive biomarkers can be developed to better predict GC prognoses and guide more effective therapeutic strategies.

\section{Disclosure}

The authors report no conflicts of interest in this work.

\section{References}

1. Venerito M, Link A, Rokkas T, Malfertheiner P. Gastric cancer-clinical and epidemiological aspects. Helicobacter. 2016;21:39-44. 
2. Wang WH, Huang JQ, Zheng GF, Lam SK, Karlberg J, Wong BC. Non-steroidal anti-inflammatory drug use and the risk of gastric cancer: a systematic review and meta-analysis. J Natl Cancer Inst. 2003; 95(23):1784-1791.

3. McMillan DC. Systemic inflammation, nutritional status and survival in patients with cancer. Curr Opin Clin Nutr Metab Care. 2009;12(3): 223-226.

4. Schreiber RD, Old LJ, Smyth MJ. Cancer immunoediting: integrating immunity's roles in cancer suppression and promotion. Science. 2011;331(6024):1565-1570.

5. Balkwill F, Mantovani A. Inflammation and cancer: back to Virchow? Lancet. 2001;357(9255):539-545.

6. Sakurai K, Ohira M, Tamura T, et al. Predictive potential of preoperative nutritional status in long-term outcome projections for patients with gastric cancer. Ann Surg Oncol. 2016;23(2):525-533.

7. Deng Q, He B, Liu X, et al. Prognostic value of pre-operative inflammatory response biomarkers in gastric cancer patients and the construction of a predictive model. J Transl Med. 2015;13:66.

8. Hirashima M, Higuchi S, Sakamoto K, Nishiyama T, Okada H. The ratio of neutrophils to lymphocytes and the phenotypes of neutrophils in patients with early gastric cancer. J Cancer Res Clin Oncol. 1998;124(6):329-334.

9. Sakurai K, Tamura T, Toyokawa T, et al. Low preoperative prognostic nutritional index predicts poor survival post-gastrectomy in elderly patients with gastric cancer. Ann Surg Oncol. 2016;23(11): 3669-3676.

10. Greiner M, Pfeiffer D, Smith RD. Principles and practical application of the receiver-operating characteristic analysis for diagnostic tests. Prev Vet Med. 2000;45(12):23-41.

11. Proctor MJ, McMillan DC, Morrison DS, Fletcher CD, Horgan PG, Clarke SJ. A derived neutrophil to lymphocyte ratio predicts survival in patients with cancer. Br J Cancer. 2012;107(4):695-699.

12. Pietrzyk L, Plewa Z, Denisow-Pietrzyk M, Zebrowski R, Torres K. Diagnostic power of blood parameters as screening markers in gastric cancer patients. Asian Pac J Cancer Prev. 2016;17(9):4433-4437.

13. Forrest LM, McMillan DC, McArdle CS, Angerson WJ, Dunlop DJ. Evaluation of cumulative prognostic scores based on the systemic inflammatory response in patients with inoperable non-small-cell lung cancer. Br J Cancer. 2003;89(6):1028-1030.

14. Wu Y, Zhou BP. Inflammation: a driving force speeds cancer metastasis. Cell Cycle. 2009;8(20):3267-3273.
15. Jung MR, Park YK, Jeong O, et al. Elevated preoperative neutrophil to lymphocyte ratio predicts poor survival following resection in late stage gastric cancer. J Surg Oncol. 2011;104(5):504-510.

16. Lee DY, Hong SW, Chang YG, Lee WY, Lee B. Clinical significance of preoperative inflammatory parameters in gastric cancer patients. $J$ Gastric Cancer. 2013;13(2):111-116.

17. Wang F, Liu ZY, Xia YY, et al. Changes in neutrophil/lymphocyte and platelet/lymphocyte ratios after chemotherapy correlate with chemotherapy response and prediction of prognosis in patients with unresectable gastric cancer. Oncol Lett. 2015;10(6):3411-3418.

18. Sun KY, Xu JB, Chen SL, et al. Novel immunological and nutritionalbased prognostic index for gastric cancer. World J Gastroenterol. 2015; 21(19):5961-5971.

19. Ji F, Liang Y, Fu SJ, et al. A novel and accurate predictor of survival for patients with hepatocellular carcinoma after surgical resection: the neutrophil to lymphocyte ratio (NLR) combined with the aspartate aminotransferase/platelet count ratio index (APRI). BMC Cancer. 2016;16:137.

20. Ardi VC, Kupriyanova TA, Deryugina EI, Quigley JP. Human neutrophils uniquely release TIMP-free MMP-9 to provide a potent catalytic stimulator of angiogenesis. Proc Natl Acad Sci U S A. 2007; 104(51):20262-20267.

21. Bambace NM, Holmes CE. The platelet contribution to cancer progression. J Thromb Haemost. 2011;9(2):237-249.

22. Milne K, Alexander C, Webb JR, et al. Absolute lymphocyte count is associated with survival in ovarian cancer independent of tumorinfiltrating lymphocytes. J Transl Med. 2012;10:33.

23. Kobayashi N, Usui S, Kikuchi S, et al. Preoperative lymphocyte count is an independent prognostic factor in node-negative non-small cell lung cancer. Lung Cancer. 2012;75(2):223-227.

24. Mantovani A, Allavena P, Sica A, Balkwill F. Cancer-related inflammation. Nature. 2008;454:436-444.

25. Kanda M, Mizuno A, Tanaka C, et al. Nutritional predictors for postoperative short-term and long-term outcomes of patients with gastric cancer. Medicine (Baltimore). 2016;95(24):3781.

26. Coussens LM, Werb Z. Inflammation and cancer. Nature. 2002; 420(6917):860-867.

27. Kim EY, Lee JW, Yoo HM, Park CH, Song KY. The platelet-tolymphocyte ratio versus neutrophil-to-lymphocyte ratio: which is better as a prognostic factor in gastric cancer? Ann Surg Oncol. 2015;22(13): $4363-4370$
OncoTargets and Therapy

\section{Publish your work in this journal}

OncoTargets and Therapy is an international, peer-reviewed, open access journal focusing on the pathological basis of all cancers, potential targets for therapy and treatment protocols employed to improve the management of cancer patients. The journal also focuses on the impact of management programs and new therapeutic agents and protocols on

\section{Dovepress}

patient perspectives such as quality of life, adherence and satisfaction The manuscript management system is completely online and includes a very quick and fair peer-review system, which is all easy to use. Visit http://www.dovepress.com/testimonials.php to read real quotes from published authors. 